logical and resource geology map to be published at 1:10,000,000 in 1981 and de Wit requested data to assist in this. Such a compilation will allow further testing of the reconstruction but it is unlikely that location of the major blocks will be in error by more than a few $100 \mathrm{~km}$. The importance of this map is thus to highlight areas for future research.

A major consideration was the extent of Gondwanaland. Nepal (C.J. Klootwyk, Canberra), Iran (H. Wensink, Utrecht) and Thailand (S. Bunopas \& P. Vella, Wellington) were formerly parts of Gondwanaland and there is evidence that much of China (J.B. Waterhouse, Queensland; P. Tasch, Wichita) and possibly some central Asian blocks, with parts of southern Europe (D.H. Tarling, Newcastle upon Tyne), also formed parts of this supercontinent at various times, the degree of floral and faunal interchange between the Gondwanan and Laurasian continents having clearly altered as both continents evolved. The distribution of dinosaurs seems to indicate that Afro-

D.H. Tarling is a Senior Lecturer in the Department of Geophysics and Planetary Physics at the University of Newcastle-uponTyne.
Indian links persisted well into Late Cretaceous times (E.H. Colbert, Flagstaff) although it is not clear if such links were provided by the Laccadive-Mascarene Ridge or whether access was along the Iranian-Afghanistan coastlines. Palaeobiogeographic provinces formed a significant part of the meeting, with the key glossopterid flora now being divisible into six stratigraphic units that have, so far, been correlated between India and Australia (J.F. Rigby, Geological Survey, Queensland; S.C. Shah, Geological Survey, Calcutta). The presentation of major advances in palynological correlations were predictable, but nonetheless of critical value for the Carbo-Permian (E.M. Truswell, BMR, Canberra) and Permo-Triassic (J. Anderson, Witwatersrand). Although this work solves many problems it also raises others; it destroys the previous, elegantly simple concept of a Gondwanaland drifting through polar regions and hence being glaciated at successive periods in different areas. The age of these ice sheet deposits are almost entirely Sakmarian (Lower Permian) and require much more complex explanations (D.H. Tarling in Climatic Change, (Ed. Gribbin) Cambridge University Press, 1978).

vibrations with zero momentum parallel to the surface, as in infrared studies.

The short range interactions are much weaker but their effects can be separated because they can transfer large amounts of momentum to the electron (Ho, Willis \& Plummer Phys. Rev. Lett. 40, 1463; 1978). Tuning the spectrometer to the nonspecular electrons immediately separates these processes, but at the price of requiring much greater sensitivity. We immediately gain two advantages: the need for a dipole moment is removed; and vibrations with non-zero parallel momentum can be seen. There is a further less obvious gain in information. The matrix element for the scattering involves an incident-wave momentum $k_{\mathrm{j}}$ an exit wave momentum $k_{\mathrm{f}}$ and the displacement of the $j$ th atom, $\mathbf{C}_{\mathrm{j}}$, caused by the vibration:

$$
\sum \int_{j} \exp \left\{\left(\mathbf{k}_{\mathrm{i}} \cdot \mathbf{r}-\mathbf{k}_{\mathrm{f}} \cdot \mathbf{r}\right)\right\} \mathbf{C}_{\mathrm{j}} \cdot \boldsymbol{\nabla} V_{\mathrm{j}}\left(\mathbf{r}-\mathbf{R}_{\mathrm{j}}\right) \mathrm{d}^{3} \mathrm{r}
$$

where $V_{\mathrm{j}}$ is the potential of the $j$ th atom, assumed localised near $\mathbf{R}_{\mathrm{j}}$. If we were investigating the modes of a molecule, it would be useful to know how $\mathbf{C}_{\mathrm{j}}$ varied within the molecule. Equation (1) shows that the contribution of each atom to the matrix element has a phase

$\exp \left\{i\left(\mathbf{k}_{\mathrm{i}}-\mathbf{k}_{\mathrm{f}}\right) \cdot \mathbf{R}_{\mathrm{j}}\right\}$

\title{
Vibrations of atoms at surfaces
}

\section{from John Pendry}

THE vibrational properties of atoms and molecules adsorbed on surfaces are determined by the detailed interaction with the surface: whether a molecule dissociates, to what degree an atom is coordinated by surface atoms, whether new species are formed. Knowledge of the way atoms vibrate is an essential complement to our static picture of atomic arrangements. The traditional tool for studying vibrations is infrared absorption, which has yielded much information about vibrations but is limited by the need for the bond to have a strong polar element to be infrared active, and in the case of metal surfaces by the electromagnetic boundary condition which allows only a normal component of the electric field. Electron energy loss spectroscopy (EELS) offers an alternative means of studying vibrational frequencies which to some extent escapes from these restrictions (Ibach Phys. Rev. Lett. 24, 1416; 1970).

Experiments make use of $1-10 \mathrm{eV}$ electrons, which can be monochromated to a few meVs. This represents much worse resolution than infrared can provide and severely restricts the power of the technique. It is unlikely that electron

John Pendry is at the SRC'S Daresbury

Laboratory. spectrometry can approach $1 \mathrm{meV}$ resolution and so the technique is mainly concerned with studying strong bonds to relatively light atoms. Despite these considerable restrictions EELS is proving a useful technique which is continually developing both experimentally and theoretically.

There are two ways in which electrons interact with vibrating atoms: through long range and short range forces. If the vibration has an associated dipole moment, an electric field extends far from the surface and operates on an approaching or receding electron for a relatively long period. If a dipole moment is present, this is the dominant mode for inelastic scattering. (Evans \& Mills Phys. Rev. B7, 853; 1973). Because the forces are long range the electron loses energy but remains undeviated in direction. A specular reflection at the surface turns the electron around into an analyser which separates the inelastic component. Until recently this has been the predominant mode of operation of EELS experiments. The requirement of a dipole moment imposes some of the restrictions of infrared spectroscopy and the lack of any substantial momentum transfer to the electron in the inelastic collision limits the information that can be obtained to therefore by measuring at different values of $\mathbf{k}_{\mathrm{i}}-\mathbf{k}_{\mathrm{f}}$ it will be possible to identify the individual components of the mode, provided only that we already know the mean positions $\mathbf{R}_{\mathrm{j}}$ of the atoms. That is, we know the phonon 'wavefunctions' as well as the energy levels.

There is an analogy with angle-resolved photoemission experiments in which the energy and momentum of an ejected photoelectron implies by conservation the energy and momentum of the original state of the electron before excitation. (Li, Tong \& Mills Phys. Rev. in the press). Alternatively knowing both the original and final electron $E$ and $\mathbf{k}$ we can infer the q and $\omega$ of the photon. If we read phonon for photon we have the EELS experiment (whether it involves the loss or gain of a phonon). The analogy extends even further: equation (1) gives the matrix element for interaction with a phonon defining atomic displacements $\mathbf{C}_{\mathrm{j}}$. The analogous matrix element for photoemission is

$$
\frac{1}{\omega_{\mathrm{j}}} \int \exp \left\{\mathrm{i}\left(\mathbf{k}_{\mathrm{i}} \cdot \mathbf{r}-\mathbf{k}_{\mathrm{f}} \cdot \mathbf{r}\right)\right\} \mathbf{A}_{\mathrm{j}} \cdot \boldsymbol{\nabla} V_{\mathrm{j}}\left(\mathrm{r}-\mathrm{R}_{\mathrm{j}}\right) \mathrm{d}^{3} \mathrm{r}
$$

where $\mathbf{A}_{\mathrm{j}}$ is the 'A vector' of the photon field at the $j$ th atom. This close analogy should lead to rapid development of the associated theory of EELS which will be needed to realise the full potential information in the data 